\title{
Calidad formal, impacto y visibilidad de las revistas electrónicas universitarias españolas
}

\author{
Por Helena Zamora, Isidro Aguillo, José Luis Ortega y Begoña Granadino
}

\begin{abstract}
Resumen: Se analizan y evalúan mediante descriptores formales e indicadores cuantitativos las revistas universitarias españolas, que se publican en formato exclusivamente electrónico. Se estudia el impacto y la visibilidad de estas publicaciones en la red. Se han estudiado tanto el número de publicaciones, como sus características formales y de contenido y sí se han adoptado las posibilidades y ventajas que aporta el medio electrónico. Los resultados son pobres, habiéndose detectado problemas formales en la edición de las sedes web de las revistas, lo que redunda en una reducida visibilidad y, por lo tanto, en un impacto muy bajo.

Palabras clave: Revistas electrónicas, Universidades españolas, Evaluación de revistas, Indicadores cibermétricos, Metodología de evaluación.

Title: Quality, impact and visibility of Spanish university electronic journals

Abstract: An analysis evaluating electronic journals published by Spanish universities was performed, taking into account both formal aspects and cybermetric indicators. The aim of this study was to analyse the impact and visibility of these web-based publications. The number of publications, their formal and content-related characteristics, and their

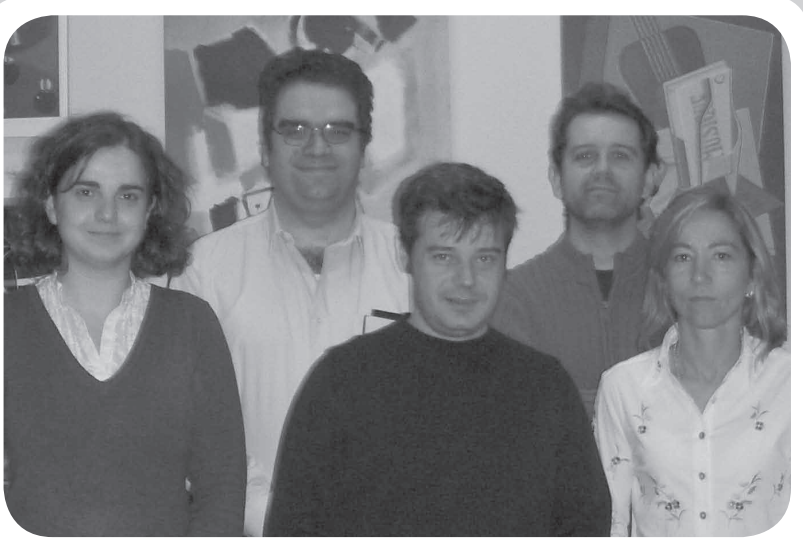

Los autores forman parte del Grupo de Investigación en Cibermetría (antiguo Internetlab) del Cindoc, dedicado al estudio cuantitativo de los procesos de comunicación científica a través de la web. Helena Zamora es documentalista con una beca de investigación para estudiar las instituciones culturales en la web. Isidro Aguillo desarrolla indicadores web para evaluación de universidades y centros de I+D. José Luis Ortega está próximo a leer su tesis doctoral sobre visualización del espacio académico europeo en la web y Begoña Granadino, científico titular del CSIC, compagina su actividad cibermétrica con otros estudios de la producción científica y tecnológica, fundamentalmente en ciencias de la vida, ciencias ambientales y biotecnología. También aparece Mario Fernández, que tiene un contrato postdoc con el Grupo.

use of features and tools provided by the electronic medium were all considered. The results fall below expectations, due to formal problems in the structure and contents of the journals' websites, resulting in low visibility and, therefore, low impact.
\end{abstract}

Keywords: Electronic journals, Spanish universities, Evaluation of scientific journals, Cybermetric indicators.

Zamora, Helena; Aguillo, Isidro; Ortega, José Luis; Granadino, Begoña. "Calidad formal, impacto y visibilidad de las revistas electrónicas universitarias españolas". En: El profesional de la información, 2007, enero-febrero, v. 16, n. 1, pp. 13-23.

http://dx.doi.org/10.3145/epi.2007.ene.02

\section{Introducción}

El sistema español de ciencia y tecnología ha experimentado un cambio fundamental en la última década debido a las decisiones estratégicas para la introducción y uso de las tecnologías de información y de la comunicación (TICs). La universidad como institución fundamental en el sistema I+D español también se ha visto influida por estos cambios. La expansión de las universidades ha contribuido al crecimiento de la actividad investigadora, aportando el $41 \%$ del personal dedicado a I+D en toda España (e-Estrategias, 2004). Esta actividad científica conlleva una gran producción de información y documentación, potenciada e incrementada por el uso de los nuevos medios de divulgación.

Uno de los canales de difusión que más se ha prosperado en la última década ha sido internet. Sus características de inmediatez, accesibilidad y economía satisfacen las nuevas necesidades de distribución de la información y el conocimiento científico, ya que facilitan la colaboración internacional en materia de in- 
vestigación que es vital para cualquier país (Thelwall; Aguillo, 2003).

La gestión de contenidos científico-técnicos es un sector estratégico para la sociedad del conocimiento y la política cultural de un país (Rodríguez Bravo, 2004). La cantidad y calidad de la información y del conocimiento que se encuentra en la web puede ser el escenario idóneo para realizar un análisis del estado de la cuestión en materia de I+D (Aguillo, 2005). De este modo, el estudio de su contenido permite saber el nivel de maduración y asimilación del conocimiento científico de un país.

Esta situación ha facilitado que tipos tradicionales de documentos se hayan pasado al ámbito electrónico, como las revistas científicas, formando parte del concepto de e-Ciencia, definido como conjunto de actividades científicas realizadas mediante recursos distribuidos accesibles a través de internet (Fecyt, 2004).

En el contexto de códigos y elementos que aporta la world wide web es necesario establecer indicadores para analizar el estado de la producción universitaria en internet y cómo ésta es eficaz para alcanzar los objetivos planteados, ya que los criterios de evaluación empleados en los documentos en formato papel son insuficientes (Arroyo, 2005).

En los últimos años se han publicado distintas opciones para la evaluación de recursos digitales en general, y de revistas electrónicas en particular. Propuestas como las establecidas por Hitchcock, Carr y Hall (1996) o la de Rodríguez Gairín (2001), basada en el estudio realizado por Codina (2000) sobre parámetros e indicadores de recursos digitales, suponen innovaciones metodológicas para el establecimiento de una serie de pautas de calidad web. También han aparecido iniciativas de evaluación de revistas científicas, alguna de los cuales, como RESH ${ }^{1}$ del Cindoc no tiene en cuenta las revistas electrónicas. Otros proyectos como los realizados por la Universidad de Barcelona $\left(M I A R^{2}\right)$, de Granada (In-Recs $\left.{ }^{3}\right)$ o la de Zaragoza $\left(E R C E^{4}\right)$ no cubren todas las áreas de conocimiento sino que se centran únicamente en revistas de las denominadas ciencias sociales y humanas.

En este estudio se pretende analizar los niveles de calidad que adquieren las revistas electrónicas universitarias españolas. Para ello se utilizan dos tipos de indicadores que se complementan entre sí:

-Cuantitativos: basados en criterios cibermétricos, definidos en el proyecto europeo Eicstes y aplicados en la iniciativa Webometrics ranking of world universities.

http://www.eicstes.org

http://www.webometrics.info
-Cualitativos o descriptivos (Aguillo, 2004), que mejoran la visión global de los resultados a través de métodos heurísticos.

\section{Metodología}

Para el presente estudio se adoptó como unidad documental básica para el análisis la sede web de las revistas electrónicas, concepto definido por Aguillo (1998) y ampliado por Arroyo y Pareja (2003), como la página o conjunto de ellas ligadas a una principal identificables por un url y que forman una unidad documental reconocible e independiente de otra, bien por su temática, por su autoría o por su representatividad institucional.

La búsqueda y localización de las revistas se realizó a través de diferentes estrategias de consulta en buscadores (Google, Yahoo!), bases de datos especializadas (Latindex, ISSN) y mediante navegación manual por las webs de las universidades españolas. Los requisitos mínimos que debían tener las publicaciones seleccionadas son: estar vinculadas a un grupo de investigación, departamento, facultad o universidad española; ser revistas electrónicas puras, es decir, editadas exclusivamente en formato digital, y poseer ISSN propio.

Se realizó una navegación completa por todas las que habían sido localizadas con el fin de comprobar su estado general y proceder al establecimiento de los puntos a evaluar. Posteriormente se constituyó una serie de indicadores cuantitativos y cualitativos que valoran los diferentes aspectos que informan de la calidad de las revistas citadas y que se utilizaron para calibrar los siguientes parámetros: tecnología, contenido y diseño.

Los datos aportados en este informe se recogieron entre los meses de julio y septiembre de 2005.

\section{"Se definieron indicadores cuantitativos y cualitativos para valorar la calidad de las revistas"}

\subsection{Datos cuantitativos}

La evaluación cuantitativa de las revistas-e se efectuó mediante indicadores cibermétricos, que aportan medidas de tres tipos: descriptivas de las sedes web, de visibilidad e impacto y de popularidad. Las herramientas utilizadas han sido:

- Bases de datos del ISI Web of knowledge: mediante su servicio Cited references se estudió el número de veces que una aportación científica es citada en 
los artículos posteriores, como medida del impacto que un trabajo ha tenido sobre la comunidad científica especializada.

- Motores de búsqueda y sus aplicaciones web, que permiten averiguar, por ejemplo, el vecindario hipertextual, es decir, la relación de las revistas con otros recursos digitales y sus patrones más habituales. Se perciben conductas que no se detectarían mediante el análisis independiente de la web de la revista. Los motores utilizados han sido Google, Yahoo! Search y MSN Search, junto con otros servicios como Google Scholar, PageRank, etc. Se han utilizado delimitadores o comandos para la obtención de información de la visibilidad hipertextual (sólo con Yahoo! Search y MSN Search pues Google no proporciona valores de enlaces a dominios) y sobre popularidad (Alexa).

- Mapeadores: Xenu's Link Sleuth para generar informes cuantitativos de los objetos informáticos que componen la sede web.

\subsection{Datos cualitativos}

Se tuvieron en cuenta criterios de arquitectura de la información, definida por Rosenfeld y Morville (1998), como la actividad dedicada a diseñar la organización, indexado, etiquetado y sistemas de navegación necesarios para la búsqueda y localización de todo sitio web. En este estudio se han englobado todos estos términos bajo el epígrafe de arquitectura de la información, adoptando el criterio establecido por BaezaYates, Rivera y Velasco (2004).

La conexión entre la extracción de la información deseada y los indicadores cualitativos necesarios originó la metodología empleada, que se sometió a los criterios de estudio de la usabilidad por inspección, es decir, la evaluación heurística, que fue definida por Nielsen como la inspección sistemática del diseño de vista del usuario. Su propósito es encontrar los problemas de usabilidad en el diseño como una parte del proceso de diseño interactivo.

Se ha caracterizado cada uno en 4 niveles como máximo, para poderlos cuantificar:

\section{"La cantidad de información que ofrece una web podría ser un indicador de su calidad (Lluís Codina)"}

\section{a. Indicadores de contenido}

Codina (2000) estableció que la cantidad de información que ofrece una sede web podría ser un indicador de su calidad y así lo apoyan y lo premian las políticas europeas, como en el caso de eContent. Los indicadores que se establecieron para evaluar el contenido de las revistas electrónicas son los siguientes:

- Autoría de los artículos, estableciendo diferentes niveles de exhaustividad. Es importante conocer este dato, así como la afiliación institucional del autor, información de contacto o su currículum personal.

- Formato de los artículos. Html, que es el característico de la web, facilita la visualización en la pantalla, pero puede presentar dificultades cuando se trata de guardar o de imprimir el texto. Otros, por el contrario, resultan más fáciles para el acceso a la información desde un punto de vista práctico y enriquecen el acceso del usuario a la revista deseada. Es el caso del pdf, que permite una gran calidad de impresión (Abadal; Rius, 2006)

- Publicación diferida: los períodos de publicación en internet deben estar sólo condicionados por el trámite que conlleva el envío de su artículo y su revisión, no debe necesitar plazos fijos para cerrar la edición de un número.

- Internacionalización: además de las implicaciones puramente lingüísticas, el idioma constituye también un factor de importancia en relación con la accesibilidad del sitio que contiene el texto, puesto que recientemente se ha comprobado que los motores de búsqueda presentan claros sesgos idiomáticos, particularmente positivos en el caso del inglés. En efecto, Aykin (2005) ha demostrado que el uso del inglés hace que la información que lo utiliza resulte más visible tanto para el usuario, como para el motor de búsqueda que lo indexa.

\section{b. Indicadores formales}

- Accesibilidad: en la evaluación de la adecuación o no de las directrices de accesibilidad establecidas por el $W 3 C$ se utilizó una herramienta online, $T A W$, que sigue las directrices de accesibilidad y está avalada por el Ministerio de Industria, Turismo y Comercio.

- Dirección de la web: la url debe identificar explícitamente la sede web que nombra y esto depende de la profundidad de la dirección url y del tipo que sea.

- Estructura de la sede web: el nivel valorado ha sido el que establece una estructura jerárquica o en árbol, por ser la más usual y la más correcta, al facilitar la navegación al usuario.

- Estadísticas de uso: aportan información sobre cantidad de visitantes, visitas, páginas vistas, vía por la que llegaron los usuarios, palabras clave utilizadas en los buscadores, las páginas de entrada, de salida, procedencia geográfica, etc. Estos datos dan información sobre la aceptación y el impacto de la revista y el posicionamiento web. 
- Mención de responsabilidad de la sede web: es necesario que se identifique el responsable de sus contenidos y conocer el organismo que avala los artículos y la autoría de la sede.

- Especificación de modelo de licencia de la propiedad intelectual de los artículos: se debe aclarar que tipo de licencia ampara la propiedad intelectual de los contenidos. El modelo habitual en estos momentos es el copyright, pero se están abriendo paso nuevos tipos como creative commons, copyleft, open access, etc.

- Consistencia en la navegación: según Fox e Instone (2001) todos los elementos que requiere una web para navegar deben ser persistentes y comunes dentro de cada sitio. Se trata de acceder en cualquier momento y del mismo modo a todas las opciones que existan dentro del sitio web considerado.

- Documentos multimedia de apoyo a la investigación: la presencia de estos materiales, tales como presentaciones de diapositivas, grabaciones de vídeo, fotografías, etc., que justifiquen, complementen o amplíen la información contenida en los artículos de la publicación.

- Navegabilidad: se analiza si la web posee un buscador, barra de navegación, mapa o cualquier otra

\begin{tabular}{|c|c|}
\hline \multicolumn{2}{|c|}{ Indicadores de las sedes web de las revistas-e } \\
\hline \multicolumn{2}{|l|}{ Cuantitativos } \\
\hline Productividad & Número de artículos por volumen y año. \\
\hline Visibilidad en bases de datos & $\begin{array}{l}\text { Presencia de los artículos en bases de datos como ISI Web of } \\
\text { knowledge o Google scholar. }\end{array}$ \\
\hline Visibilidad en motores de búsqueda & Análisis de la importancia de la web por el PageRank. \\
\hline Vecindario hipertextual & Relación entre las páginas web visitadas por los usuarios. \\
\hline Tamaño de las sedes web & $\begin{array}{l}\text { Número total de páginas de una web y tipos de formatos utiliza- } \\
\text { dos. }\end{array}$ \\
\hline Visibilidad hipertextual & $\begin{array}{l}\text { Análisis y comparación de los datos obtenidos mediante dos he- } \\
\text { rramientas: Yahoo! Search y MSN Seach. }\end{array}$ \\
\hline \multicolumn{2}{|l|}{ Cualitativos } \\
\hline \multicolumn{2}{|l|}{ a) De contenido } \\
\hline Autoría de los artículos & Información sobre los autores. \\
\hline Formato de los artículos & $\begin{array}{l}\text { Uso de los formatos que permite el medio digital en los artículos } \\
\text { (pdf, html, etc.). }\end{array}$ \\
\hline Publicación diferida & Análisis de la inmediatez de la publicación de los artículos. \\
\hline Internacionalización & $\begin{array}{l}\text { Idioma en el que aparecen los artículos publicados en las revistas, } \\
\text { publicación en un solo idioma, en varios o versiones bilingües. }\end{array}$ \\
\hline \multicolumn{2}{|l|}{ b) Formales } \\
\hline Accesibilidad & $\begin{array}{l}\text { Nivel de cumplimiento de las directrices WAI de accesibilidad en } \\
\text { las sedes web. }\end{array}$ \\
\hline Dirección de la web & Análisis de la url, profundidad y dominio propio. \\
\hline Estructura de la web & $\begin{array}{l}\text { Análisis de la estructura de la url de la sede web; relación entre } \\
\text { las urls de las diferentes páginas. }\end{array}$ \\
\hline Estadísticas de uso & Utilización y publicación de estadísticas de uso en las sedes web. \\
\hline $\begin{array}{l}\text { Mención de responsabilidad de la sede } \\
\text { web }\end{array}$ & Presencia o no. \\
\hline $\begin{array}{l}\text { Especificación del modelo de licencia de } \\
\text { la propiedad intelectual de los artículos }\end{array}$ & Presencia o no. \\
\hline $\begin{array}{l}\text { Documentos multimedia de apoyo a la } \\
\text { investigación }\end{array}$ & $\begin{array}{l}\text { Presencia de documentos multimedia que apoyen, justifiquen, } \\
\text { complementen o amplíen la información contenida en los artícu- } \\
\text { los. }\end{array}$ \\
\hline Navegabilidad & $\begin{array}{l}\text { Utilización de elementos que faciliten la navegación en una sede } \\
\text { web, como buscadores, barras de navegación, mapas, etc. }\end{array}$ \\
\hline $\begin{array}{l}\text { Contenido semántico del título de la } \\
\text { sede web }\end{array}$ & $\begin{array}{l}\text { Empleo de títulos descriptivos que permitan establecer el criterio } \\
\text { de la página, guardarla en bookmarks, etc. }\end{array}$ \\
\hline $\begin{array}{l}\text { Servicios y contenidos de valor añadi- } \\
\text { do }\end{array}$ & $\begin{array}{l}\text { Presencia de información de valor añadido, retroalimentación, ín- } \\
\text { dices, alertas, identificadores persistentes, etc. }\end{array}$ \\
\hline
\end{tabular}

Tabla 1: Indicadores cuantitativos y cualitativos aplicados a las revistas. 
herramienta que facilite la navegación y la localización de información dentro de la misma.

- Contenido semántico del título de la sede web: el uso de títulos descriptivos es un elemento importante de la usabilidad (Nielsen, 2005), pues permite a las personas que visitan una página establecer su contexto, guardarla con un nombre apropiado en sus bookmarks, etc. Incluso en algunos motores de búsqueda se le da mucha importancia a las palabras del título a la hora del posicionamiento (Baeza-Yates; Castillo; López, 2005).

- Servicios y contenidos de valor añadido: se han tenido en cuenta determinados servicios y contenidos de valor añadido que se consideran relevantes para este tipo de publicación, como son: información complementaria, retroalimentación, índices, alertas o identificadores persistentes.

\section{Resultados}

\subsection{Descripción de la población}

Según la base de datos Latindex, las revistas universitarias suponen un 45,3\% (451 revistas universitarias) del total en España. De éstas, un 23,6\% son electróni- cas, aunque únicamente un 9,3\% de las revistas universitarias españolas corresponden a revistas electrónicas puras, es decir, sólo accesibles en formato digital.

Se ha recogido una población para este estudio de 140 títulos que pertenecen a 39 de las 72 universidades españolas, lo que significa un $54,1 \%$ del total. Las que más publicaciones científicas electrónicas presentan son la Universidad Complutense de Madrid, con un 13,6\% de las revistas, y la Universidad de Barcelona y la de Valencia con un 8,6\% cada una (tabla 2).

Por número de revistas, las comunidades autónomas que destacan son Madrid (36), Cataluña (30) y Andalucía (23). Pero calculando la densidad por área geográfica sobresalen Cataluña $(4,80)$, Comunidad Valenciana $(5,00)$ y Madrid $(4,50)$ como las más productivas. Por tipología de institución son las universidades públicas, de educación presencial y multidisciplinares las que más revistas científicas electrónicas publican, frente a las universidades privadas, de educación a distancia y politécnicas. Este tipo de publicaciones son más habituales en determinados ámbitos científicos, como en ciencias sociales y humanas, destacando dentro de estos dos grupos, las publicaciones sobre pedagogía, lingüística y derecho (tabla 3 ).

\begin{tabular}{|c|c|c|c|}
\hline Universidad & $\%$ revistas & Universidad & $\%$ revistas \\
\hline Complutense de Madrid & 13,6 & Pompeu Fabra & 2,1 \\
\hline Barcelona & 8,6 & UNED (Educ. Distancia) & 2,1 \\
\hline Valencia & 8,6 & Deusto & 1,4 \\
\hline Autónoma de Barcelona & 5,7 & Islas Baleares & 1,4 \\
\hline Granada & 5,0 & Jaume I & 1,4 \\
\hline Oberta de Catalunya & 4,3 & País Vasco & 1,4 \\
\hline Sevilla & 3,6 & Salamanca & 1,4 \\
\hline Alfonso X El Sabio & 2,9 & Vigo & 1,4 \\
\hline Autónoma de Madrid & 2,9 & Zaragoza & 1,4 \\
\hline Carlos III de Madrid & 2,9 & Alcalá & 0,7 \\
\hline Murcia & 2,9 & Cantabria & 0,7 \\
\hline Oviedo & 2,9 & Cardenal Herrera & 0,7 \\
\hline Cádiz & 2,1 & Europea de Madrid & 0,7 \\
\hline Castilla la Mancha & 2,1 & Extremadura & 0,7 \\
\hline Huelva & 2,1 & Gran Canarias & 0,7 \\
\hline La Laguna & 2,1 & Jaén & 0,7 \\
\hline La Rioja & 2,1 & Juan Carlos I & 0,7 \\
\hline Málaga & 2,1 & León & 0,7 \\
\hline Politécnica de Madrid & 2,1 & Rovira i Virgili & 0,7 \\
\hline
\end{tabular}

Tabla 2: Porcentaje de revistas electrónicas por universidad 


\begin{tabular}{|l|c|}
\hline Códigos Unesco & \% revistas \\
\hline Pedagogía & 15,7 \\
\hline Lingüística & 14,3 \\
\hline Derecho & 12,1 \\
\hline CC. de las artes y las letras & 9,3 \\
\hline CC. médicas & 7,1 \\
\hline Historia & 7,1 \\
\hline CC. económicas & 5,0 \\
\hline CC. políticas & 5,0 \\
\hline CC. tecnológicas & 5,0 \\
\hline Sociología & 4,3 \\
\hline Geografía & 3,6 \\
\hline Psicología & 3,6 \\
\hline CC. de la Tierra & 2,9 \\
\hline Antropología & 1,4 \\
\hline CC. de la vida & 1,4 \\
\hline Filosofía & 0,7 \\
\hline Matemáticas & 0,7 \\
\hline Multidisciplinar & 0,7 \\
\hline
\end{tabular}

Tabla 3: Porcentaje de revistas por disciplinas

\subsection{Indicadores cuantitativos}

- Productividad de las revistas: el incremento de las publicaciones que nacen cada año ha sido continuado, con pequeñas oscilaciones a lo largo de los últimos años (figura 1). De cualquier forma tienen en su mayoría una productividad muy baja, con un número de artículos muy reducido por año, el 25\% tiene una productividad de entre 0 y 5 . En el otro lado está el 2,14\% de las revistas que tiene una producción muy alta, entre 49 y 108 artículos. Esto supone una gran dispersión de los datos de productividad, la moda del número de artículos por revista está entre 26 y 40, en un 30\% de las revistas analizadas.

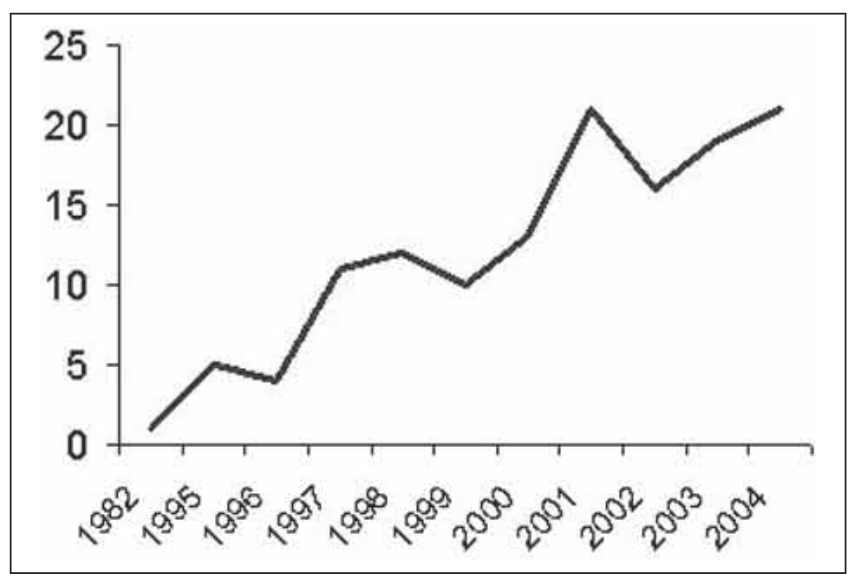

Figura 1. Años de nacimiento de las revistas electrónicas
$\mathrm{Al}$ analizar la fecha de actualización de las revistas se aprecia que el $70 \%$ de las mismas continúan ininterrumpidamente publicándose hasta el año 2005, lo cual significa que poseen una actualización adecuada.

- Visibilidad en bases de datos:

1. ISI Web of knowledge: únicamente el $15 \%$ de las revistas electrónicas de las universidades españolas aparecen citadas en la base de datos del ISI, y reciben un bajo número de citas (figura 2).

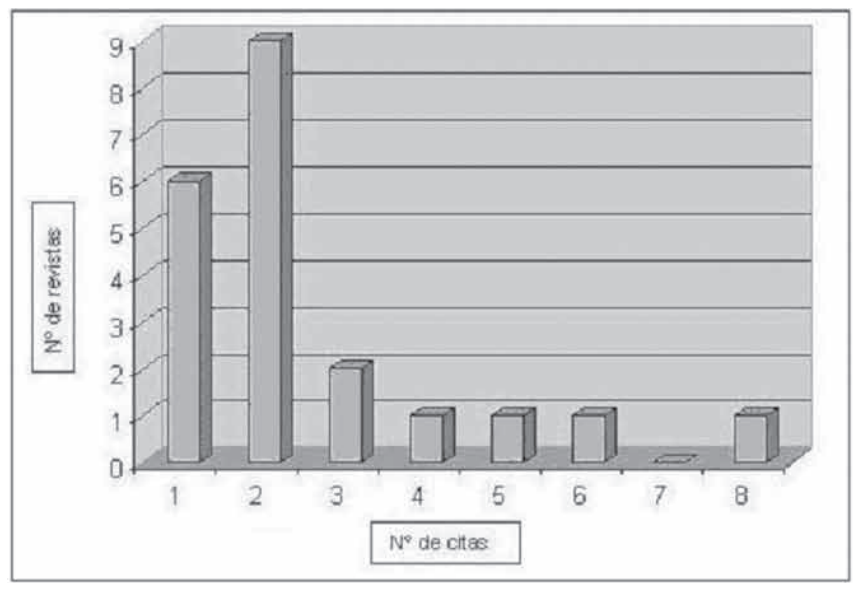

Figura 2. Número de citas recibidas en el ISI

La comparación entre el número de artículos por revista y las citas recibidas por éstos en la base de datos ISI proporcionan una aceptable coincidencia, es decir, que en la mayoría de los casos las más citadas concuerdan con las que contienen mayor número de artículos.

2. Google scholar indexa documentación técnica y científica. Sólo un $6 \%$ de las revistas analizadas supera las 11 citas; el resto no llega a esta cifra.

— Visibilidad en motores de búsqueda: PageRank es una familia de algoritmos utilizada por Google para representar la importancia que una página tiene en internet en forma de un valor numérico en una escala comprendida entre 0 y 10. En el caso de las revistas electrónicas analizadas, el valor que más se repite es el 5 , en un total de 47 casos. Sin embargo, ninguna supera la puntuación de 6 . El 85\% tienen una puntuación que oscila entre 3 y 6 , o sea que la gran mayoría tiene una puntuación intermedia de PageRank.

- Vecindario: se analizó el patrón de visitas de una web para comprobar qué páginas visitan los usuarios de las revistas electrónicas que se estaban analizando y observar las relaciones entre sedes. Se han diferenciado 4 tipos de relaciones: dependencia institucional, temática, lingüística y tipología documental. La relación más común es la lingüística, seguida por la temática (figura 3). 


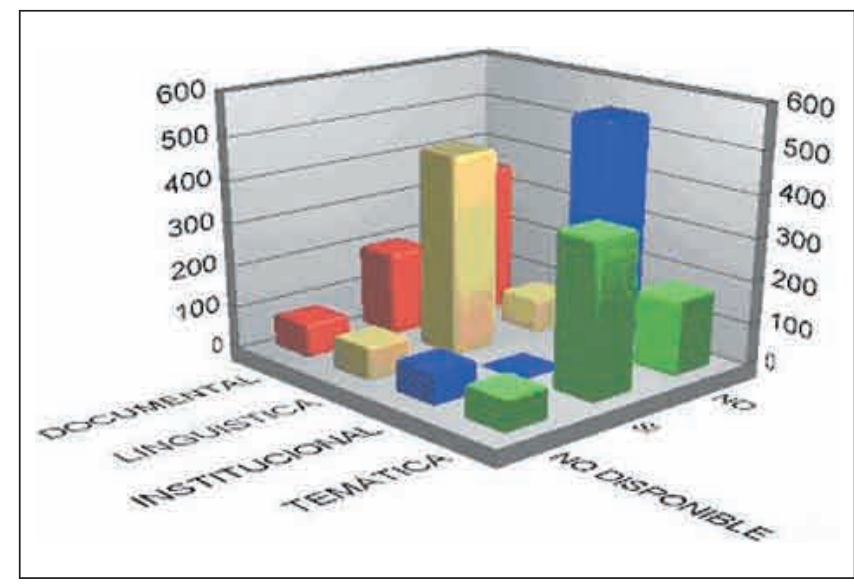

Figura 3. Vecindario hipertextual de las revistas electrónicas

- Tamaño de la web: la mayoría de las revistas estudiadas (59\%) contienen menos de 100 páginas en su sede, sólo un $4 \%$ tiene más de 1.000 . La gran mayoría (74\%) se encuentra en html, mientras que únicamente un $26 \%$ de ellas son visibles en formatos ricos, básicamente en pdf.

- Visibilidad hipertextual: consiste en el número de enlaces recibidos por una web, lo que se interpreta como una medida de impacto. El hecho de que una web sea enlazada por otra se entiende, en términos de cita hipertextual, que es similar a la cita bibliográfica en literatura impresa, por lo que este dato es de gran importancia a la hora de analizar el impacto del contenido de una revista electrónica. En este caso se han utilizado tanto Yahoo! Search como MSN Search (tabla 4).

En la tabla se recogen las 10 revistas más enlazadas, ordenadas de forma descendente por la suma de los parámetros que proporcionan los dos buscadores empleados. En primer lugar aparece Espéculo, que está

\begin{tabular}{|l|r|r|}
\hline Título de la revista & Yahoo! & MSN \\
\hline Espéculo & 1.648 & 1.129 \\
\hline $\begin{array}{l}\text { Boletín CF+S: ciudades para } \\
\text { un futuro más sostenible }\end{array}$ & 1.968 & 10 \\
\hline Hipertext.net & 1.195 & 520 \\
\hline Lamusa & 170 & 1.245 \\
\hline Medio ambiente \& derecho & 433 & 789 \\
\hline $\begin{array}{l}\text { Interactive educational } \\
\text { multimedia }\end{array}$ & 400 & 382 \\
\hline $\begin{array}{l}\text { Revista latina de } \\
\text { comunicación social }\end{array}$ & 380 & 363 \\
\hline $\begin{array}{l}\text { Relieve: revista electrónica } \\
\text { de investigación y evaluación } \\
\text { educativa }\end{array}$ & 388 & 333 \\
\hline Anglogermánica online & 45 & 556 \\
\hline $\begin{array}{l}\text { Lemir: Literatura española } \\
\text { medieval y del renacimiento }\end{array}$ & 88 & 417 \\
\hline
\end{tabular}

Tabla 4: Ranking con las 10 revistas con mayor visibilidad hipertextual situada muy arriba de la tabla por ambos, sin embargo, hay revistas que están muy bien situadas en uno y muy mal en el otro.

\subsection{Indicadores cualitativos}

\subsubsection{Indicadores de contenido}

- Autoría de los artículos: el 75\% de los trabajos analizados únicamente indican el nombre de los autores y algún otro dato de localización de los mismos. Tan sólo un 7\% de las revistas electrónicas aporta otros datos de identificación, de contacto o un breve currículum vitae de los autores (figura 4).

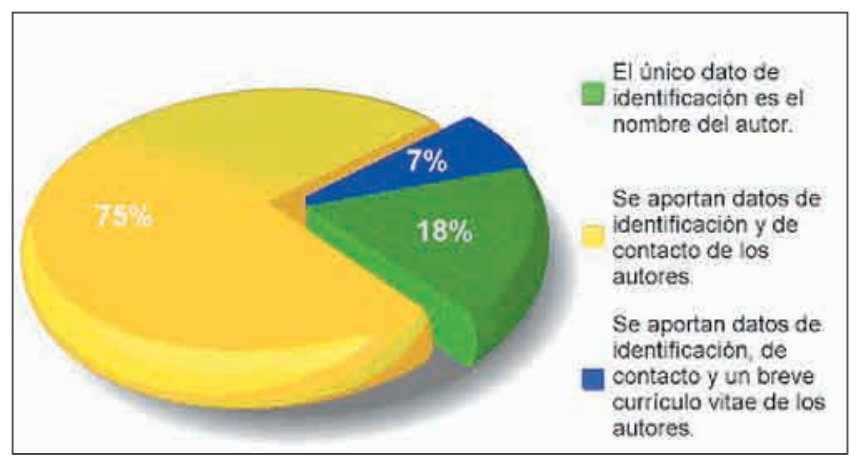

Figura 4. Revistas por nivel de identificación del autor

- Formato de los artículos: el 16\% de las publicaciones tiene acceso a los documentos exclusivamente en html. El 50\% de las revistas tienen sus artículos accesibles únicamente en formatos ricos, generalmente en pdf. Tan sólo un 34\% permite elegir a los usuarios entre formatos ricos y html.

- Publicación diferida: del total de revistas que integran la población estudiada para este informe, únicamente un $16 \%$ publica sus artículos de forma inmediata.

- Multilingüísmo: el 8\% de las revistas analizadas posee versiones completas de al menos una tercera parte los documentos en otros idiomas, excepto en inglés. Y tan sólo un $9 \%$ contiene una versión en inglés de los documentos a texto completo (figura 5).

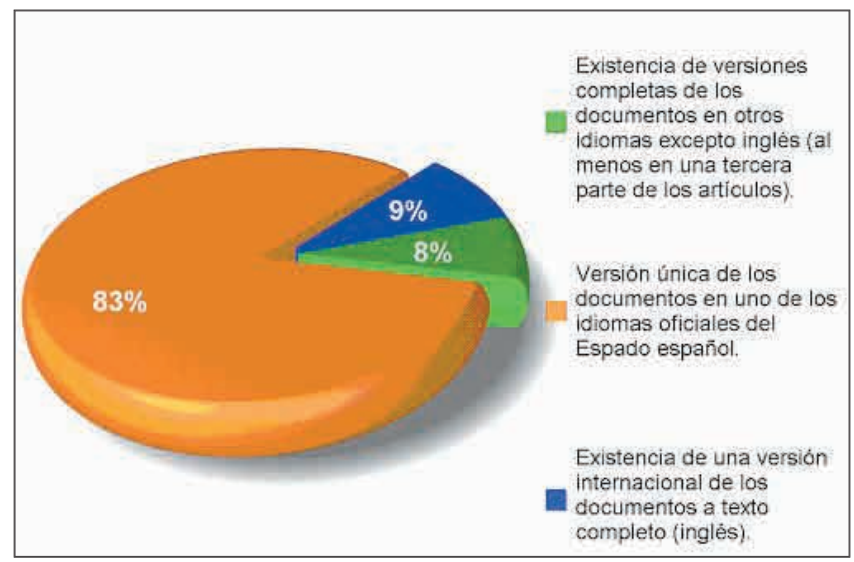

Figura 5. Multilingüísmo de los artículos 


\subsubsection{Indicadores formales}

- Accesibilidad: En la evaluación de la adecuación o no de las directrices de accesibilidad establecidas por el $W 3 C$ se utilizó $T A W^{5}$. El $82 \%$ de las sedes web de las revistas analizadas no se ajusta a ningún criterio WAI de accesibilidad, el $6 \%$ cumple únicamente el primer nivel de accesibilidad WAI(A) y tan sólo un $11 \%$ verifica el nivel $A A$ (figura 6).

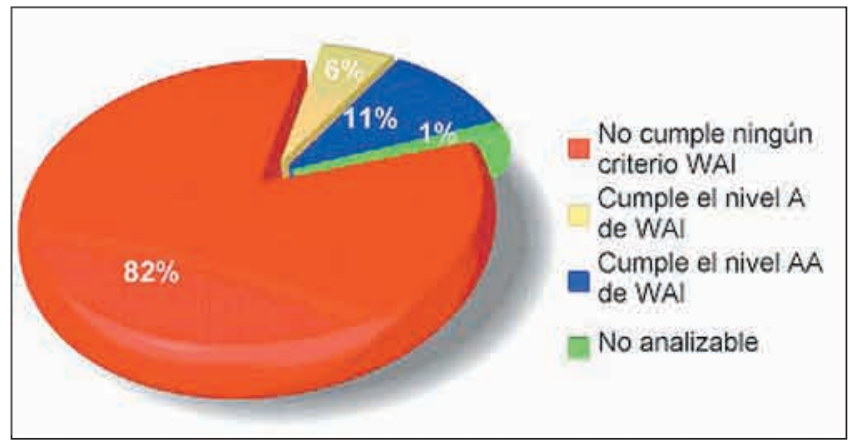

Figura 6. Niveles de accesibilidad de las revistas-e

\section{"El $82 \%$ de las webs de las revistas analizadas no cumple ningún criterio WAl de accesibilidad"}

— Dirección de la sede web: únicamente un $6 \%$ de las revistas-e posee un dominio propio, lo cual facilita su localización y su visibilidad. Un dato muy negativo es el hecho de que la mayoría de las revistas, un 58\%, tiene una dirección con dos o más niveles, lo que repercute en la visibilidad de las mismas (figura 7).

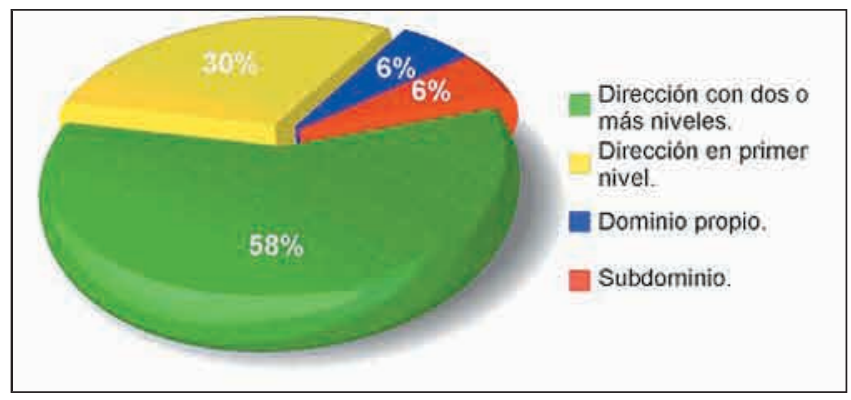

Figura 7. Dirección de las webs de las revistas-e

- Estructura de la sede web: la mejor valorada ha sido la estructura jerárquica o en árbol que es utilizada por el $47 \%$. Sin embargo, en el $51 \%$ no existe una correcta relación jerárquica entre las páginas (figura 8).

- Estadísticas de uso: este servicio es importante por la información que aporta sobre aceptación, impacto y posicionamiento web, pero sólo un $16 \%$ cuenta con él.

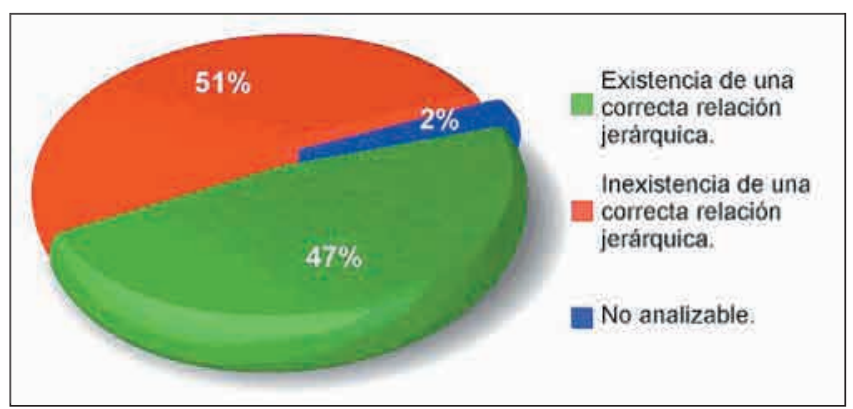

Figura 8. Estructura de las webs de las revistas-e

- Mención de responsabilidad de la sede web: en el $39 \%$ de los casos aparece en la página de inicio, en el $41 \%$ es visible desde cualquier página y en el $19 \%$ no se encuentra.

- Especificación del modelo de licencia de la propiedad intelectual de los artículos: se indica en el $44 \%$ de las revistas, siendo el más habitual el copyright.

- Consistencia en la navegación: un $41 \%$ de las revistas presenta un diseño lógico y consistente que facilita la navegación a través de su contenido. El 33\% muestran un diseño coherente que aparece a lo largo de toda la sede web, de modo que se consigue una homogeneidad que facilita su uso. Finalmente, un $26 \%$ acusa un diseño carente de consistencia (figura 9).

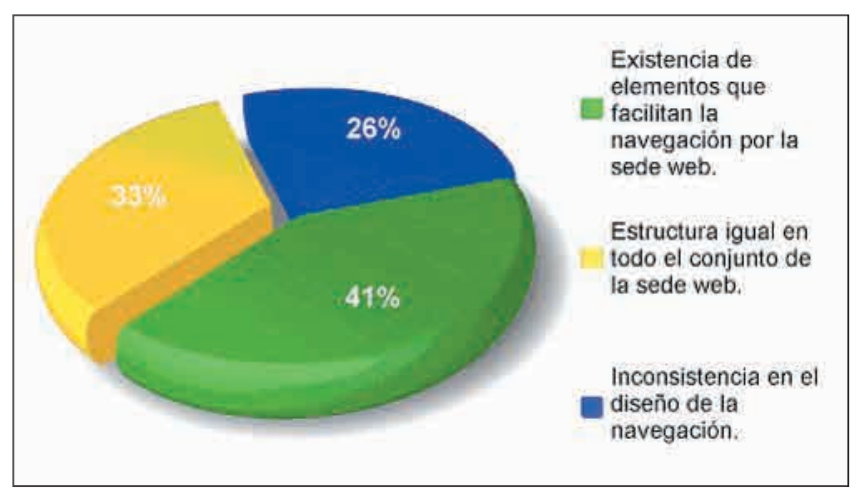

Figura 9. Consistencia en la navegación de las webs de las revistas-e

- Documentos multimedia de apoyo a la investigación: la gran mayoría de los casos, un 95\%, no los utiliza, pese a que el entorno digital favorece su uso.

- Navegabilidad: El 76\% de las revistas cuentan con la existencia de, al menos, un elemento que facilite la navegabilidad, ya sea un menú categorizado, una barra de navegación, un motor de búsqueda o cualquier otra utilidad. El $24 \%$ carece de todo dispositivo a tal efecto.

- Contenido semántico del título de la sede web: en una gran mayoría, el 77\%, existe correspondencia semántica entre este dato y el contenido, mientras que en un $23 \%$ el título no se ajusta al material ofrecido. 


\section{baratz}

En primera línea en

Sistemas de Información y Gestión del Conocimiento
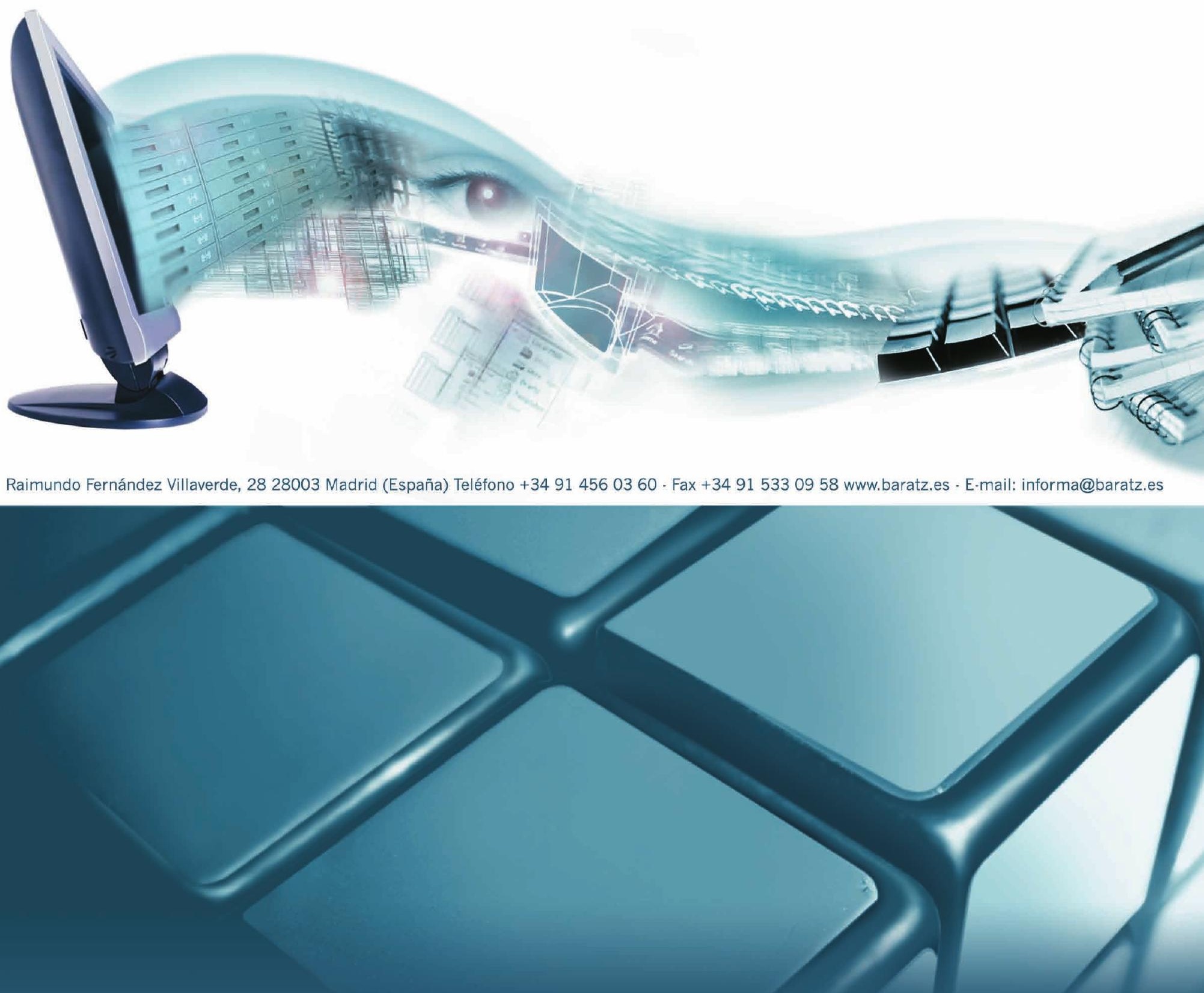

(2) Soluciones para bibliotecas: Absys, absysNET, Absys

(9) Gestión documental y del conocimiento: BKM, Baratz Windows, BRSCGI

(2) Soluciones para archivos: Albalá

(2) Servicios de Catalogación Retrospectiva

(2) Edición en CD-Rom

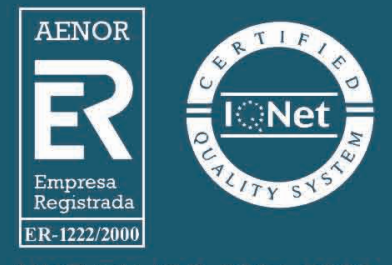


- Servicios y contenidos de valor añadido: en un $71 \%$ se saca partido del carácter electrónico de la revista y se ofrecen alertas, identificadores persistentes, información añadida, retroalimentación, etc., lo que incide en el aumento del valor de la publicación.

\section{Discusión y conclusiones}

Un primer análisis de las revistas muestra unos resultados con un patrón difuso, debido posiblemente al bajo tamaño de la población, pero que es coherente con lo que cabría esperar. Destacan las universidades madrileñas, catalanas, andaluzas y valencianas, son más productivas las públicas que las privadas, y la contribución de las politécnicas y las de enseñanza a distancia es reducida. Temáticamente, las humanidades y las ciencias sociales explotan especialmente la web como canal de comunicación, destacando el número de títulos de pedagogía, lingüística y derecho.

El estudio del impacto y la visibilidad de la literatura científica muestra que son muy pocas las revistas analizadas que han sido citadas por las bases de datos Thomson ISI, lo que da una medida clara del poco impacto que tienen todavía las revistas electrónicas en general, y las editadas por las universidades españolas en particular.

El análisis del patrón de visitas de los usuarios confirma que el acceso a la información en internet se realiza mayoritariamente a través de motores de búsqueda. Utilizando buscadores especializados las relaciones temáticas y lingüísticas generadas a partir de patrones de visita se correlacionan con los resultados de búsquedas temáticas en los motores de búsqueda.
En los datos cuantitativos sobre las características principales de las webs, como pueden ser su tamaño (número y tipología de los objetos informáticos y documentales), riqueza hipertextual (enlaces y su distribución), etc., destaca el pequeño tamaño en número de páginas que contienen. También muestran una escasa proporción de archivos en formatos ricos o documentos multimedia, que facilitan el acceso a la información desde un punto de vista práctico (unidades documentales), y que enriquecen y complementan el acceso del usuario a la información.

Uno de los aspectos negativos son los graves problemas formales de las sedes web, que redundan en la baja visibilidad y que dificultan de forma considerable el acceso a los contenidos de las revistas electrónicas tanto de los usuarios finales como de los robots de los motores de búsqueda. Muchas sedes tienen direcciones complejas, con urls excesivamente largas y/o poco descriptivas, los mecanismos de navegación son confusos o incluso opacos a los motores, y en bastantes casos las estructuras no son jerárquicas. La responsabilidad última de este aspecto podría corresponder a las propias universidades e indica la falta de un compromiso claro de las mismas hacia la edición de revistas específicamente electrónicas.

Las sedes analizadas no cumplen en su mayoría con ninguno de los criterios de accesibilidad. La falta de una adecuación generalizada a las directrices recogidas explícitamente en la legislación vigente, perjudica el acceso a la información.

Otros aspectos formales también aparecen descuidados, a veces como resultado de la mera transposición de las normas habituales en papel, que resultan insufi-

\section{Próximos temas centrales Marzo 2007 Web 2.0: blogs, participación y Lib 2.0 Mayo 2007 Portales y gestión de contenidos (CMS) Julio 2007 Bibliotecas universitarias Septiembre 2007 Documentación multimedia Noviembre 2007 Ontologías}

Los interesados pueden remitir notas, artículos, propuestas, publicidad, comentarios, etc., sobre estos temas a: epi@elprofesionaldelainformacion.com 
cientes cuando no pobres en el entorno electrónico. La ausencia de enlaces a páginas personales en la autoría, la dilación innecesaria para cumplir periodicidades, la no disponibilidad de versiones en formatos alternativos o en otros idiomas o la falta de acceso a estadísticas de consumo son algunos de los más destacados.

En términos generales se puede indicar que la cantidad de revistas electrónicas universitarias españolas es baja y, salvo contadas excepciones, no forma parte del eje central de la comunicación científica en nuestro país. Ello resulta paradójico ya que los datos muestran un impacto destacable en la web de los títulos electrónicos, debido a su mayor audiencia potencial como a las novedosas posibilidades que ofrece el soporte. Lamentablemente la adopción de dichas innovaciones, tanto formales como de contenido, no se ha generalizado entre los títulos estudiados.

Este trabajo ha sido financiado por el Ministerio de Educación y Ciencia bajo el Programa de Estudios y análisis, convocatoria de 2005 .

http://www.mec.es/univ/proyectos2005/EA20050008.pdf

\section{Notas}

1. http://resh.cindoc.csic.es/

2. http://bd.ub.es/miar/default.php

3. http://ec3.ugr.es/in-recs/

4. http://erce.unizar.es/

5. http://www.tawdis.net/

\section{Bibliografía}

Abadal, E.; Rius, L. "Revistas científicas digitales: características e indicadores". En: Revista de universidad y sociedad del conocimiento, 2006, v. 3, n. 1, p. 620 .

Aguillo, I. F. "Hacia un concepto documental de sede web". En: El profe sional de la información, 1998, v. 7, n. 1-2, pp. 45-46.

Aguillo, I. F., et al. "What the internet says about science". En: The scientist, 2005, v. 19, n. 14, p. 10.

Aguillo, I. F., et al. Factor de impacto y visibilidad de 4.000 sedes web universitarias españolas. Cindoc-CSIC, 2004. Consultado en: 30-05-06. http://www.cindoc.csic.es/estudios_ea2004_0020_informe.doc

Aguillo, I. F. Cibermetría: la métrica de la web [seminario], 2003. Consultado en: 30-05-06.

http://www.archivovirtual.org/seminario/busqueda/ponencias/p2.htm

Arroyo, N., et al. "Cibermetría. Estado de la cuestión”. En: 9as Jornadas españolas de documentación, 2005, pp. 273-290.

Arroyo, N.; Pareja, V. M.; Aguillo, I. F. Description of web data in D3.1. Eicstes project, deliverable 3.2, 2003. Consultado en: 25-05-06. http://www.eicstes.org/

Aykin, N. Usability and internationalization of information technology. Mahwah: Lawrence Erlbaum Associates. 2005. ISBN 0805844791.

Baeza-Yates, R.; Rivera, C.; Velasco, J. "Arquitectura y usabilidad en la web”. En: El profesional de la información, 2004, v. 13, n. 3, pp. 168-178.

\section{¿Recibes ya E P \\ en casa?}

Por sólo 75 euros

$+$

$4 \%$ IVA

puedes tener todos los meses tu copia particular y leerla cómodamente cuando quieras.

Codina, L. "Parámetros e indicadores de calidad para la evaluación de recursos digitales". En: 7as Jornadas españolas de documentación, 2000, pp. $135-144$.

Fecyt. Libro blanco e-Ciencia en España 2004. Consultado en: 15-05-06. http://www.fecyt.es/documentos/e-Ciencia.pdf

Hitchcock, S.; Carr, L.; Hall, W. Making the most of electronic journals, 1998. Consultado en: 23-05-06.

http://xxx.lanl.gov/html/cs.DL/9812016

Laboratorio de internet. Webometrics ranking of world universities. Consultado en: 01-05-06.

http://www.webometrics.info/

Nielsen, J.; Mack, R. L. Usability inspection methods. New York: John Wiley \& Sons, 1994. ISBN 0-471-01877-5.

Rodríguez Bravo, B. Evaluación del acceso y gestión de los libros electrónicos en las bibliotecas universitarias españolas. Ministerio de Educación y Ciencia, 2004. Consultado en: 15-12-05.

http://wwwn.mec.es/univ/html/informes/estudios_analisis/resultados_2004/ ea0115/ea0115.pdf

Rodríguez Gairín, J. M. "Parámetros e indicadores de calidad en la evaluación de una revista electrónica. El caso de BiD: textos universitaris de biblioteconomia i documentació". En: Revista de biblioteconomia i documentació, 2001, junio, n. 6.

Rosenfeld, L.; Morville, P. Information architecture for the world wide web. Cambridge: O'Reilly, 1998. ISBN 1-56592-282-4.

Thelwall, M.; Aguillo, I. F. "La salud de las web universitarias españolas". En: Revista española de documentación científica, 2003, v. 26, n. 3, pp. 291-305.

Helena Zamora, Isidro F. Aguillo, José Luis Ortega y Begoña Granadino, Grupo de Investigación en Cibermetría, Cindoc-CSIC, Joaquín Costa, 22. 28002

Madrid, España.

hzamora@cindoc.csic.es

isidro@cindoc.csic.es

jortega@cindoc.csic.es

bgranadino@cindoc.csic.es 